\title{
The Construction of Recombinant D6 Clone for in Vitro Breast Cancer Study
}

\author{
Tan Wee Yee, Khoo Boon Yin, and Chew Ai Lan
}

\begin{abstract}
D6, which is also known as CCBP 2, is one of the decoy chemokine receptors. It was recently found to play a role in the progression of breast cancer cells. In this study, the existence of D6 in invasive breast cancer cells, MDA-MB-231 was investigated by One-step RT-PCR with additional Pfu DNA polymerase in the reaction. The amplicons were then sequenced and compared with the reference sequence from GenBank database. Nucleotide sequence analysis showed that the amplicon sequence matches the reference sequence. Thus, it is confirmed that full length D6 sequence had been amplified from MDA-MB-231.
\end{abstract}

Index Terms-Cloning, D6, DNA sequencing, MDA-MB-231.

\section{INTRODUCTION}

Breast cancer is the most common cancer in women in most parts of the world. Majority of the breast cancer patients were found to surrender to this disease due to cancer invasion and metastasis [1]. In Oncology study, breast cancer research had become one of the most progressively evolving fields [2]. To date, with the advanced understanding of key molecular features, breast cancer is no longer considered a single disease but a combination of different subtypes with different biological behaviors and clinical outcomes [3]. Novel molecules and new diagnostic methods are being discovered and developed constantly, globally. Recently, the identification of various signaling pathways implicated in the cellular process of breast cancer cells has drawn the attention of researchers worldwide. The involvement of growth factors or signaling molecules in breast cancer cell proliferation and invasion were reported worldwide [4]-[7]. For examples, chemokines and chemokine receptors were reported to be involved in cancer growth and metastasis [8]-[13].

D6 (or CCBP2) was originally known as a binding molecule of CCL3 and its' expression was found in murine hemopoietic stem cells [14]. However the expression of D6 in human cells was found to be atypical, in which it will not lead to the activation of signaling pathway upon binding with the respective target proteins [1]-[16]. The findings engrossed the attention of oncologist and researchers to investigate the roles of D6 in cancer networks. Recent findings showed that D6 is plentiful on different types of

Manuscript received February 8, 2013; revised April 19, 2013

This work was funded by the Exploratory Research Grant Scheme (ERGS) from the Ministry of Higher Education Malaysia under grant 203/ CIPPM/ 6730059.

Tan Wee Yee, Chew Ai Lan, amd Khoo Boon Yin are with the Institute for Research in Molecular Medicine (INFORMM), Universiti Sains Malaysia, 11800 USM, Penang, Malaysia (e-mail: wyee129@ yahoo.com, boonyin@usm.my, chew@usm.my; ai_lan@hotmail.com). human cells such as leukocytes, lymphatic endothelial cells of the gut and lungs, malignant vascular tumors, to name a few [1]-[18]. Besides, the involvement of D6 in breast cancer has been described in recent years too [8]-[16].

In the present study, the presence of D6 in invasive breast cancer cells, MDA-MB-231 was investigated. The nucleotide sequence of D6 was amplified and recombinant clone of D6 was constructed.

\section{Methodology}

\section{A. Maintenance of MDA-MB-231 Cell Line}

MDA-MB-231 (ATCC number: HTB-26), which is a highly invasive human breast cancer cell line, was routinely maintained in DMEM (Dulbecco's modified Eagle's medium) supplemented with $10 \%$ heat inactivated fetal bovine serum (FBS), $100 \mathrm{U} / \mathrm{ml}$ of penicillin and $100 \mu \mathrm{g} / \mathrm{ml}$ of streptomycin. The cells were grown and maintained in a humidified 5\% carbon dioxide incubator. The cells were then used for total RNA extraction.

\section{B. Total RNA Extraction}

The $80 \%$ confluent cultured cells were trypsinized and the cell pellet was obtained by centrifugation using a table top centrifuge. Then, total RNA was purified from the cultured cells using commercially available RNA extraction kit (AxyPrep Multisource Total RNA Miniprep kit, Axygen). The purification of total RNA was carried out according to the user manual provided by the kit's manufacturer.

\section{Generation of Full Length D6 Sequence}

Total RNA purified from the previous step was used as a template in the One-step RT-PCR. To perform this, Maxime RT-PCR Premix kit (Intron) was used to convert total RNA to cDNA and then PCR, continuously in a single tube reaction. For proof-reading purpose, appropriate amount of Pfu DNA polymerase (BioAtlas) was manually added into the premix reaction mix prior to the experiment. Genespecific primers as mentioned below (Table I) were used in the PCR reaction. Published sequence of D6 with GenBank accession number NM_001296.4 was used as reference sequence for the generation of gene specific primers. Different restriction sites were incorporated in the primer sequences, to allow the ligation of PCR product into desired expression vector. Forward primer contains EcoRI restriction site (bolded) whereas reverse primer contains restriction site for SacII (bolded). One-step RT-PCR was started with 30 minutes hot start at $45^{\circ} \mathrm{C}$ in a $20 \mu 1$ reaction. Then, cDNA samples were denatured at $94^{\circ} \mathrm{C}$ for 5 minutes. After that, the reaction was cycled 35 times at $94^{\circ} \mathrm{C}$ for 30 seconds, annealing at $61^{\circ} \mathrm{C}$ for 30 seconds and elongation at 
$72^{\circ} \mathrm{C}$ for 2 minutes. Finally, the reaction was extended at $72^{\circ} \mathrm{C}$ for 10 minutes towards the end of PCR. Negative control reaction consisted of everything as mentioned in the sample reaction except total RNA purified from the cell lines. The PCR products were electrophoresed on $1 \%$ agarose gel stained with ethidium bromide, and visualized under UV light. DNA Ladder, 100 bp Plus (Fermentas) was used as a marker.

TABLE I: THE GENE-SPECIFIC PRIMERS USED IN THIS STUDY

\begin{tabular}{llc}
\hline \hline Primer & Sequence (5'-3') & Tm \\
& & \\
\hline Forward & GCGAATTCGCATTTCCTTCCAACATGGCC & 63.04 \\
Reverse & GCCCGCGGAGGCTGATTTATTCCCCACATCC & 67.27 \\
\hline
\end{tabular}

\section{TA Cloning and DNA Sequencing}

Gel purification kit (Fermentas) was used to purify PCR products from the agarose gel after 60 minutes of $100 \mathrm{~V}$ gel electrophoresis. Ligation of gel purified sample into TA vector, pTZ57R/T (Fermentas), was performed at $20^{\circ} \mathrm{C}$ using T4 Ligase (Fermentas). The ligation process was terminated by heat inactivating the samples at $65^{\circ} \mathrm{C}$ for 10 minutes, after 2 hours of incubation. Upon ligation, recombinant vectors were transformed into electrocompetent bacterial cells, TOP 10 (Invitrogen). The electrocompetent cells with $2 \mu \mathrm{l}$ of recombinant vectors were pulsed in an electroporation cuvette $(0.2 \mathrm{~cm})$ using an electroporator (Bio-Rad). Cells were revived immediately by the addition of $1 \mathrm{ml} \mathrm{LB}$ broth after pulsing. Transformed cells were agitated for 30 minutes at $37^{\circ} \mathrm{C}, 750 \mathrm{rpm}$ using a thermoblock shaker before plating on a low salt LB agar (with $25 \mu \mathrm{g} / \mathrm{ml}$ Zeocin, Invitrogen) and incubated at $37^{\circ} \mathrm{C}$, overnight to select positive clones. Selected positive clones were propagated overnight in a Zeocin containing low salt LB broth. Plasmid extraction kit (Fermentas) was used to purify plasmid DNA from the clones. Then, EcoRI and SacII (Fermentas) were used in the double digestion analysis The presence of full length sequence of interest gene in the clones was confirmed on $1 \%$ agarose gel stained with ethidium bromide after double digestion process. Positive clones carrying the gene of interest were sent for sequencing Primers used for the sequencing were universal primers, M13F_20 and M13R_pUC_26. Nucleotide sequence from GenBank with the accession number of NM_001296.4 was used as a reference and the sequencing result was analysed by ClustalW.

\section{E. Cloning of D6 Nucleotide Sequence into Yeast Expression Vector}

Positive TA clones carrying the gene of interest were restricted at EcoRI and SacII sites based on the double digestion protocol from Fermentas. The digested recombinant TA vectors were gel purified prior to further experiments. Yeast expression vector, pPICZ alpha A (Invitrogen) was used as eukaryote expression vector in this study. The agarose gel purified gene of interest was ligated into pPICZ alpha A by T4 Ligase (Fermentas) based on the ligation protocol as described above. The recombinant yeast expression vectors were then electro-transformed into electrocompetent TOP 10 for propagation purpose. The transformed TOP 10 were screened again by plate selection method, and positive clones were selected for plasmid purification. The positive recombinant plasmid DNA was sent for sequencing and analysed again by ClustalW. Sequencing of recombinant yeast expression vector was performed using forward AOX1 primer and reverse AOX1 primer.

\section{RESULTS AND DISCUSSION}

The presence of D6 in invasive breast cancer cells was an attractive topic since atypical chemokine receptors had started to gain attention and interest in recent breast cancer studies [16]-[20]. Hence, MDA-MB-231, which is an invasive breast cancer cell line, was chosen in this study, and the existence of D6 in the cell line was investigated.

\section{A. Total RNA Extraction}

The total RNA was extracted using Axygen kit and separated by agarose gel electrophoresis. Fig. 1 showed the total RNA extracted from MDA-MB-231 which consisted of 28s rRNA, 18s rRNA, 5s rRNA and tRNA.

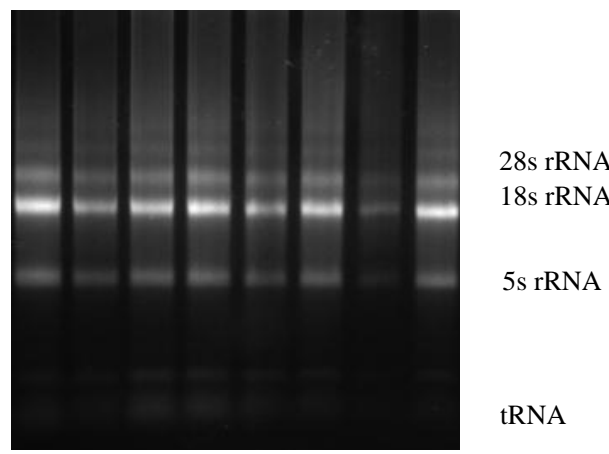

Fig. 1. The gel picture of total RNA extracted from MDA-MB-231. The distinct bands of $28 \mathrm{~s}$ rRNA, 18s rRNA, $5 \mathrm{~s}$ rRNA and tRNA in the total RNA were clearly shown.

\section{B. Generation of Full Length D6 Sequence}

Total RNA from MDA-MBA-231 was reversetranscribed and full length D6 sequence was amplified in a single tube reaction using gene-specific primer pairs. Forward primer was built to hook the nucleotides from 165 $\mathrm{bp}$ to $185 \mathrm{bp}$ on the reference sequence (NM_001296.4), whereas reverse primer was built to flank the nucleotides from 1310 bp until 1331 bp on the same sequence. Reverse primer was designed to allow in frame cloning of interest gene (insert) without stop codon, with myc and His tag at the $\mathrm{C}$ terminal to be cloned in the yeast expression vector. Based on the position of gene-specific primer pairs on reference sequence from GenBank, the amplified PCR product of One-step RT-PCR was expected to be in the range of 1185-1300 bp with the additional of restriction enzymes (EcoRI and SacII) recognition sites and multiple A nucleotides at the $\mathrm{C}$ terminal. Fig. 2 showed the amplified full length D6 sequence on ethidium bromide stained $1 \%$ agarose gel. The amplicon band was observed to be approximately $1200 \mathrm{bp}$ based on 100 bp Plus DNA Ladder (Fermentas). The location of the bands on gel picture was correspondent to the estimated size of full length D6 sequence, indicating existence of D6 in MDA-MB-231.

\section{TA Cloning and DNA Sequencing}

Upon TA cloning, double digestion was performed to 
confirm the presence of interest gene in the TA vector. Full length D6 sequence with the size approximately $1200 \mathrm{bp}$ was shown on $1 \%$ agarose gel after the overnight incubation of TA clones with specific restriction enzymes (Fig. 3). The band appeared within the range of 2000-3000 bp was correspondent to the size of empty TA vector, which is 2886 bp (Fermentas); while another band appeared above 3000 bp was expected to be undigested recombinant TA vector, which harbours the full length sequence of D6. The result showed the success of TA cloning and the positive clone was then sent for DNA sequencing.

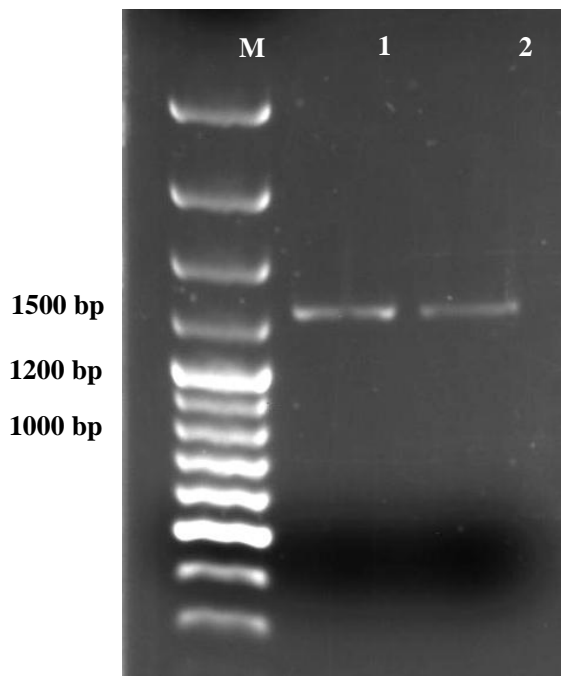

Fig. 2. The amplicons at the size of approximately $1200 \mathrm{bp}$, were shown on ethidium bromide stained $1 \%$ agarose gel. $\mathrm{M}$ lane refers to $100 \mathrm{bp}$ Plus DNA ladder (Fermentas). Lanes 1 and 2 refer to One-Step RT-PCR products, with total RNA extract from MDA-MB-231.

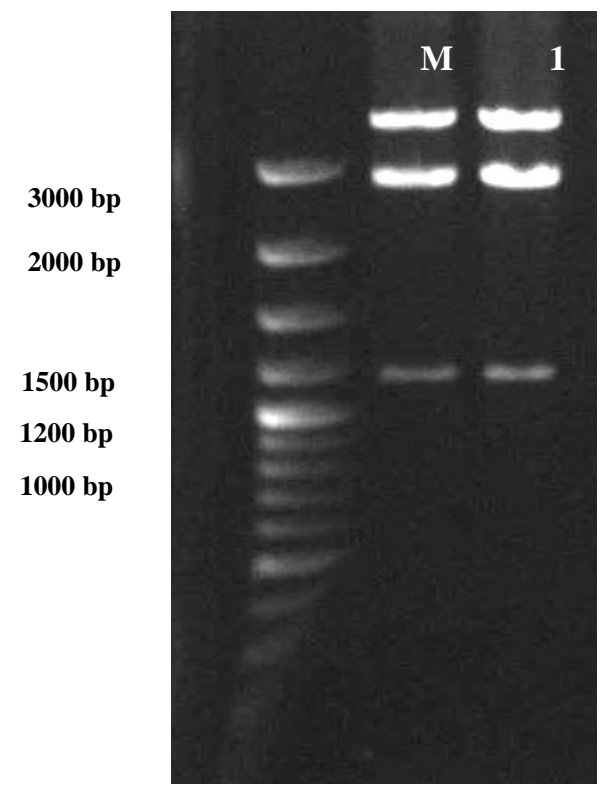

Fig. 3. Double digestion was performed to confirm the ligation of full length D6 sequence in TA vector. M refers to 100 bp Plus DNA Ladder (Fermentas), whereas Lanes 1 and 2 refer to recombinant TA vectors which were double digested with EcoRI and SacII.

Sanger sequencing was known to be accurate in DNA sequencing compared to other method such as Maxam Gilbert method [21]. It is a DNA sequencing method with the use of chain terminating inhibitors [22]. However, to sequence the full length of sample DNA, two reactions were performed under Sanger sequencing method. Forward reaction with forward primer M13F_20 and reverse reaction with reverse primer M13R_pUC_26 were carried out. ClustalW analysis was performed by aligning sample sequence with the reference sequence, D6 (NM_001296.4) from GenBank. The analysis showed no mismatch, deletion or insertion in amplified sequence compared to the reference sequence (Result not shown). DNA sequence of amplicon matched reference sequence from GenBank database. Hence, the finding deduced that a complete full length D6 sequence of MDA-MB-231 had been amplified using One-step RTPCR.

\section{Cloning of D6 Nucleotide Sequence into Yeast Expression Vector}

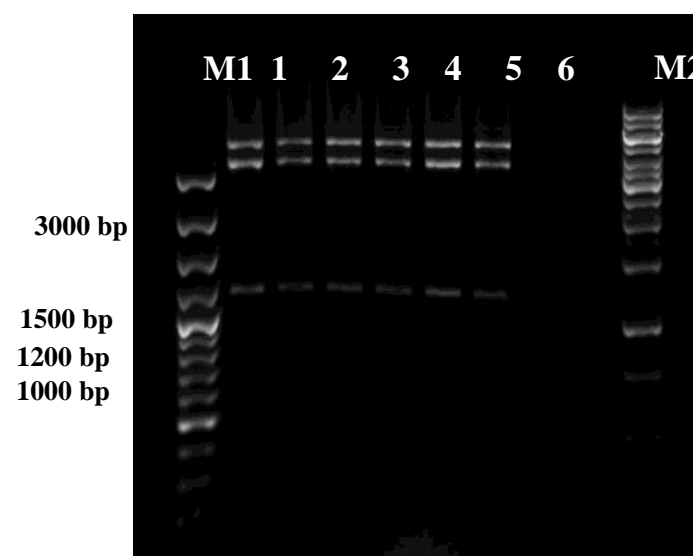

Fig. 4. The double digestion of recombinant pPICZ alpha A vector. M1 refers to 100 bp Plus DNA Ladder whereas M2 refers to $1 \mathrm{~kb}$ DNA Ladder (Fermentas). Lanes 1 to lane 6 refer to the digestion of recombinant pPICZ alpha A vector.

Yeast expression vector (Invitrogen), pPICZ alpha A is $3593 \mathrm{bp}$ in length. Agarose gel picture showed that the full length sequence of D6 was cloned into the expression vector (Fig. 4). Gel picture showed double digestion yielded three distinct bands. The band of $1200 \mathrm{bp}$ was the gene of interest, whereas another two bands appeared above 3000 bp were digested and undigested pPICZ alpha A, respectively.

wo reactions of Sanger DNA sequencing were performed again to read the full length sequence of the insert; one with forward AOX1 primer while another with reverse AOX1 primer. ClustalW analysis showed a complete matching of insert sequence from the recombinant yeast expression vector to the reference sequence from GenBank database. Besides, the sequencing reactions with forward AOX1 and reverse AOX1 primers also showed that the gene of interest was embedded in-frame and downstream of alpha factor signal sequence and in-frame with $c$-myc and polyhistidine tag in yeast expression vector. Hence, the gene can be expressed as a recombinant fusion protein.

\section{CONCLUSION}

Current study showed that One-step RT-PCR with the aid of Pfu DNA polymerase amplified potential D6 sequence from total RNA of MDA-MB-231. The DNA sequencing with the aid of two different sequencing primers further proved the presence and also confirmed the nucleotide sequence of full length D6 in MDA-MB-231 cell lines. The generated recombinant D6 clones will be used in-house for further experiments in breast cancer studies. The research 
outcome will serve as a catalyst to channel rapid and innovative advances of our research findings to the biotechnology industry.

\section{ACKNOWLEDGEMENTS}

The first author would like to thank National Science Fellowship from Ministry of Science, Technology and Innovation, Malaysia for the scholarship awarded during the tenureship.

\section{REFERENCES}

[1] C. A. Lan, T. W. Yee, and K. B. Yin, "Potential combinatorial effects of recombinant atypical chemokine receptors in breast cancer cell invasion: A research perspective (Review)," Biomedical Reports, January 21, 2013, vol. 1, pp. 185-192.

[2] T. Vora, E. de Azambuja, A. Awada, and M. Piccart, "Novel therapeutics in breast cancer--Looking to the future," Update on Cancer Therapeutics, 2009, vol. 3, pp. 189-205.

[3] R. Sandhu, J. S. Parker, W. D. Jones, C. A. Livasy, and W. B. Coleman, "Microarray-Based Gene Expression Profiling for Molecular Classification of Breast Cancer and Identification of New Targets for Therapy," Lab Medicine, vol. 41, pp. 364-372.

[4] E. F. Adams, B. Rafferty, and M. C. White, "Interleukin-6 is secreted by breast fibroblasts and stimulates 17beta-oxidoreductase activity in MCF-7 breast cancer cells: possible paracrine regulation of $17 \mathrm{beta}-$ oestradiol levels," Int J Cancer, vol. 49, pp. 118-121, 1991.

[5] J. Adnane, P. Gaudray, and C. Dionne, "BEK and FLG, two receptors to members of the FGF family, are amplified in subsets of human breast cancers," Oncogene, 1991, vol. 6, pp. 659-663.

[6] F. K. Ahmad, S. Deris, and M. S. Abdullah, "Synergy network based inference for breast cancer metastasis," Procedia Computer Science, vol. 3, pp. 1094-1100.

[7] N. Cabioglu, A. A. Sahin, P. Morandi, F. Meric-Bernstam, R. Islam, H. Y. Lin, and C. D. Bucana, "Gonzalez-Angulo AM, Hortobagyi GN, Cristofanilli M. Chemokine receptors in advanced breast cancer: differential expression in metastatic disease sites with diagnostic and therapeutic implications," Annals of Oncology, 2009, vol. 20, pp. 1013-1019.

[8] X.-H. Zeng, Z.-L. Ou, K.-D. Yu, L.-Y. Feng, W.-J. Yin, J. Li, Z.-Z. Shen, and Z.-M. Shao, "Coexpression of atypical chemokine binders (ACBs) in breast cancer predicts better outcomes," Breast Cancer Research and Treatment, 2011, vol. 125, pp. 715-727.

[9] X. Tang, Z. Sun, C. Runne, J. Madsen, F. Domann, M. Henry, F. Lin, and S. Chen, "A critical role of Gbetagamma in tumorigenesis and metastasis of breast cancer," Journal of Biological Chemistry, vol. 286, pp. 13244-13254, 2011

[10] C. Addison, J. Belperio, M. Burdick, and R. Strieter, "Overexpression of the duffy antigen receptor for chemokines (DARC) by NSCLC tumor cells results in increased tumor necrosis," BMC Cancer, 2004, vol. 4, pp. 28.

[11] S. Ali and G. Lazennec, "Chemokines: novel targets for breast cancer metastasis," Cancer and Metastasis Reviews, 2007, vol. 26, pp. 401420.

[12] P. Allavena, G. Germano, F. Marchesi, and A. Mantovani, "Chemokines in cancer related inflammation," Experimental Cell Research, vol. 317, pp. 664-673.

[13] F. Balkwill, "Cancer and the chemokine network," Nature, 2004, vol. 4, pp. 540-550.

[14] M. Locati et al., "Silent chemoattractant receptors: D6 as a decoy and scavenger receptor for inflammatory CC chemokines," Cytokine \& Growth Factor Reviews, 2005, vol. 16, pp. 679-686.

[15] A. Mantovani, M. Locati, A. Vecchi, S. Sozzani, and P. Allavena, "Decoy receptors: a strategy to regulate inflammatory cytokines and chemokines," Trends in Immunology, 2001, vol. 22, pp. 328-336.

[16] F.-Y. Wu, Z.-L. Ou, L.-Y. Feng, J.-M. Luo, L.-P. Wang, Z.-Z. Shen, and Z.-M. Shao, "Chemokine Decoy Receptor D6 Plays a Negative Role in Human Breast Cancer," Molecular Cancer Research, 2008, vol. 6, pp. 1276-1288.

[17] R. J. B. Nibbs et al., "The [beta]-Chemokine Receptor D6 Is Expressed by Lymphatic Endothelium and a Subset of Vascular Tumors," The American Journal of Pathology, 2001, vol. 158, pp. 867-877.

[18] A. Mantovani, B. Savino, M. Locati, L. Zammataro, P. Allavena, and R. Bonecchi, "The chemokine system in cancer biology and therapy," Cytokine \& Growth Factor Reviews, 2010, vol. 21, pp. 27-39.
[19] L.-Y. Feng, Z.-L. Ou, F.-Y. Wu, Z.-Z. Shen, and Z.-M. Shao, "Involvement of a Novel Chemokine Decoy Receptor CCX-CKR in Breast Cancer Growth, Metastasis and Patient Survival," Clinical Cancer Research, 2009, vol. 15, pp. 2962-2970.

[20] J. Wang, Z. L. Ou, Y. F. Hou, J. M. Luo, Z. Z. Shen, J. Ding, and Z. M. Shao, "Enhanced expression of Duffy antigen receptor for chemokines by breast cancer cells attenuates growth and metastasis potential," Oncogene, 2006, vol. 25, pp. 7201-7211.

[21] F. Sakamoto, E. Suzuki, and Y. Fujii, "Novel approach for the effective determination of DNA scission site using the Sanger method," Journal of Biochemical and Biophysical Methods, vol. 52, pp. 97-109, 2002

[22] F. Sanger, S. Nicklen, and A. R. Coulson, "DNA sequencing with chain-terminating inhibitors," in Proc. the National Academy of Sciences, 1977, vol. 74, pp. 5463-5467.

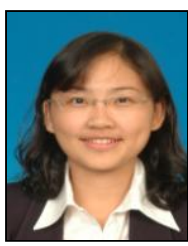

Tan Wee Yee comes from Malaysia, who obtained a bachelor degree in biotechnology from AIMST University (Kedah, Malaysia), and followed by a master degree in biotechnology from Universiti Sains Malaysia (Penang, Malaysia). Wee Yee is now a third year PhD research student in Institute for research in molecular medicine (INFORMM) in Universiti Sains Malaysia.

She had her industrial training in Universiti Putra Malaysia during her first degree. Then, she worked as a Graduate Assistant in School of Biological Sciences during her Master degree, in Universiti Sains Malaysia. She published a research paper on 'Process parameters influencing tannase production by Aspergillus niger using mangrove (Rhizophora apiculata) bark in solid substrate fermentation' in African Journal of Biotechnology in year 2011.

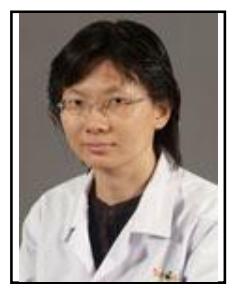

Khoo Boon Yin obtained her B.Sc. in biotechnology from Universiti Sains Malaysia in 1999. After gaining an experience in industry as a Microbiologist, she pursued her M.Sc. in molecular biology, at the same university, and obtained her $\mathrm{PhD}$ in medical biotechnology at the Health Campus of the university in 2007.

She worked as a temporary lecturer in INFORMM later. Dr. Khoo pursued her post-doctoral training at the Division of Haematology\& Oncology, Charité Campus Mitte in Berlin, Germany, funded by the Ernst-von-Leyden Scholarship, Berliner Krebsgesellschaft in 2009. She returned to USM in 2010 and continued her journey as a INFORMM's academician until now. Dr. Khoo's research interest focuses on various aspects: 1 . Development of the conventional and quantitative PCR approaches for the detection of microorganisms; 2. Understanding the interaction of mesenchymal stem cells and human breast cancer cells; and 3. Evaluation of the effects of potential novel anticancer agents, especially on anti-invasion, in highly metastatic human breast cancer cells. She establishes her research to recombinant protein production as research reagents for cancer diagnostics and therapeutics using recombinant DNA technology recently. Her research findings have been published as research articles in various international journals. In 2012, she published her first research finding on CC-chemokine family in MDA-MB231 as a book chapter in a book of breast cancer. The book chapter has achieved impressive readership results and is accessed 1500 times so far.

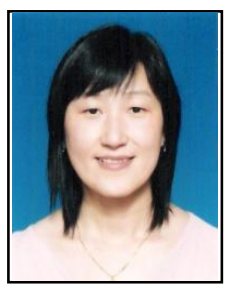

Chew Ai Lan obtained her BSc Hons majoring in microbiology from Universiti Sains Malaysia (USM) in 1997 and PhD in Biotechnology (USM) in 2005

She started her career in 2002 as a Research Scientist in a well established food and feed manufacturing company. Her work then involved the study on treatment of food and feed ingredients, agro and industrial waste via submerged and solid state fermentation technology before the value added products were put into trials and commercialization. Since joining the academic world in 2005, she has immersed herself in research subjects of industrial interest including screening and study of bioactive compounds from natural resources such as microbes and plants and production of recombinant proteins for various applications. She specializes in fermentation and enzyme technology with research interest in industrial biotechnology. She is now working as a Senior Lecturer in Institute for research in Molecular Medicine (INFORMM, USM) and her research focuses on design and development of microbial cell factories for biomolecules production. She is involved in the development and improvement of strains (bacteria, yeast and fungi) and biotechnology processes to improve yield in the production of commercially valuable products including enzymes and other proteins for laboratory and industrial applications. 